\title{
Model predictive control embedding a parallel hybrid modeling strategy
}

\author{
Debanjan Ghosh ${ }^{1}$, Jesús Moreira ${ }^{2}$, and Prashant Mhaskar ${ }^{1}$ \\ ${ }^{1}$ Department of Chemical Engineering, McMaster University, Hamilton, Ontario, \\ Canada, L8S $4 \mathrm{~L} 7$ \\ ${ }^{2}$ Imperial Oil, 505 Quarry Park Blvd, Calgary, AB T2C 5N1 \\ ${ }^{1}$ Email : mhaskar@mcmaster.ca
}




\section{Supporting Information for Publication}

Table S1: Seeded batch crystallizer parameters

\begin{tabular}{|ll|}
\hline \multicolumn{2}{|c|}{ Parameter nomenclature } \\
\hline$C:$ Solute concentration & $T:$ Reaction temperature \\
$C_{m}:$ Metastable concentration & $C_{s}:$ Saturation concentration \\
$\mu_{i}:$ ith moment of the particle size distribution & $T_{j k}:$ Jacket temperature \\
$\mu_{i}^{n}:$ ith moment corresponding to nucleation & $\rho:$ Density of crystals \\
$\mu_{i}^{s}:$ ith moment corresponding to seed/desired & $k_{v}:$ Volumetric shape factor \\
$U:$ Overall heat transfer co-efficient & $A:$ Total heat transfer surface area \\
$M:$ Mass of solvent in crystallizer & $C_{p}:$ Heat capacity of solution \\
$\Delta H:$ Heat of reaction & $E_{b}:$ Nucleation activation energy \\
$E_{g}:$ Growth activation energy & \\
\hline
\end{tabular}

Table S2: Seeded batch crystallizer parameter values and simulation settings

\begin{tabular}{ll}
\hline Parameters & Nominal Values \\
\hline$b$ & 1.45 \\
$g$ & 1.5 \\
$k_{b}$ & $285 \mathrm{~s}^{-1} \mu \mathrm{m}^{-3}$ \\
$k_{g}$ & $1.44 \mathrm{e} 8 \mu \mathrm{m} / \mathrm{s}$ \\
$\rho$ & $2.66 \mathrm{e}-12 \mathrm{~g} / \mathrm{m}^{3}$ \\
$U A$ & $0.8 \mathrm{KJ} \mathrm{s} \mathrm{K}$ \\
$k_{v}$ & 1.5 \\
$C_{p}$ & $3.8 \mathrm{KJ} /(\mathrm{Kg} \mathrm{K})$ \\
$\Delta H$ & $44.5 \mathrm{KJ} / \mathrm{Kg}$ \\
$E_{b} / R$ & $7517 \mathrm{~K}$ \\
$E_{g} / R$ & $4859 \mathrm{~K}$ \\
$M$ & 27 \\
$d t$ & $3.005 \mathrm{~seconds}$ \\
$T(0)$ & $50^{\circ} \mathrm{C}$ \\
$C(0)$ & $0.1742 \mathrm{~g} / \mathrm{g}$ \\
\hline
\end{tabular}


Table S3: Erroneous parameters in the first principles model (original values of these parameters are listed in Table S2p.

\begin{tabular}{cc}
\hline Parameter & \% Mismatch \\
\hline$E_{b} / R$ & 0.8 \\
$E_{g} / R$ & 0.8 \\
$g$ & 10 \\
$b$ & 10 \\
\hline
\end{tabular}

Table S4: Batch initial conditions and noise parameters

\begin{tabular}{ccc}
\hline Variable & $\sigma_{\text {batchwise }}$ & $\sigma_{\text {noise }}$ \\
\hline$n$ & 0.2 & 0.05 \\
$C$ & 0.0028 & 0.008 \\
$T$ & 0.52 & 0.003 \\
$\mu_{i}^{n}, \mu_{i}^{s}(i=1,2,3)$ & - & 0.001 \\
\hline
\end{tabular}

Table S5: Model Identification database summary

\begin{tabular}{ccc}
\hline Input policy & Set-point profile & Number of Batches \\
\hline PI trajectory tracking $\left(K_{C}=0.85, T_{I}=38\right)$ & 1 & 30 \\
PI trajectory tracking $\left(K_{C}=0.85, T_{I}=38\right)$ & 2 & 10 \\
\hline Total batches & & 40 \\
\hline
\end{tabular}

Table S6: Model and parameters

\begin{tabular}{ccc}
\hline Model & Hankel rows & Model order \\
\hline Hybrid-1,2: sfp model & 34 & 30 \\
Hybrid-1: residual model & 61 & 57 \\
Hybrid-2: residual model & 64 & 60 \\
Non-linear hybrid: Residual model & 64 & 60 \\
Subspace model & 59 & 55 \\
\hline
\end{tabular}

Table S7: Optimization Parameters

\begin{tabular}{cccc}
\hline Parameters & Case -1 & Case-2 & Case-3 \\
\hline$\delta$ & $2^{\circ} \mathrm{C} / \min$ & $2^{\circ} \mathrm{C} / \mathrm{min}$ & $2^{\circ} \mathrm{C} / \mathrm{min}$ \\
$\gamma$ & $9.930 e 9$ & $1.093 e 10$ & $1.133 e 10$ \\
\hline
\end{tabular}


MPC tuning parameter values (Implemented as per the different layers of optimization)

$$
\begin{aligned}
& \epsilon_{1}= \begin{cases}0.0035 \mathrm{~g} / \mathrm{g}, & \text { from } 10-22.5 \mathrm{~min} \\
0.0015 \mathrm{~g} / \mathrm{g} & \text { from } 22.5-30 \mathrm{~min}\end{cases} \\
& \epsilon_{2}= \begin{cases}-0.0035 \mathrm{~g} / \mathrm{g}, & \text { from } 10-22.5 \mathrm{~min} \\
-0.0015 \mathrm{~g} / \mathrm{g}, & \text { from } 22.5-30 \mathrm{~min}\end{cases} \\
& \epsilon_{3}=0.4 e 9, \text { from } 10-30 \mathrm{~min}
\end{aligned}
$$

Table S8: Kalman tuning parameters used in the state estimation during MPC implementation for the 3 different MPC's

\begin{tabular}{cccccccccccccccc}
\hline MPC - type & Model & Process noise $\left(\sigma^{2}\right)$ & \multicolumn{11}{c}{ Measurement noise $\left(\sigma^{2}\right)$} \\
\hline \multirow{2}{*}{ Hybrid-1 } & sfp & $1 e-6$ & 0.01 & 0.01 & 0.01 & 0.01 & 0.01 & 0.01 & 0.01 & 0.01 & 0.003 & 0.1 & 0.1 & 0.1 \\
& res & $1 e-4$ & 0.01 & 0.01 & 0.01 & 0.01 & 0.01 & 0.01 & 0.01 & 0.01 & 0.003 & 0.1 & 0.1 & 0.1 \\
& Sybrid-2 & $1 e-6$ & 0.01 & 0.01 & 0.01 & 0.01 & 0.01 & 0.01 & 0.01 & 0.01 & 0.003 & 0.1 & 0.1 & 0.1 \\
& sfp & $1 e-4$ & 0.01 & 0.01 & 0.01 & 0.01 & 0.01 & 0.01 & 0.01 & 0.01 & 0.003 & 0.1 & 0.1 & 0.1 \\
Subid & Subspace & $1 e-4$ & 0.01 & 0.01 & 0.01 & 0.01 & 0.01 & 0.01 & 0.01 & 0.01 & 0.003 & 0.1 & 0.1 & 0.1 \\
\hline
\end{tabular}

